\section{Qualidade da atenção básica mediante internações evitáveis no Sul do Brasil}

\author{
Quality of primary care as measured by \\ preventable hospitalizations in the South of Brazil
}

\author{
1 Faculdade de Medicina, \\ Universidade Federal de \\ Pelotas, Pelotas, Brasil. \\ 2 Programa de Pós-gradu- \\ ação em Saúde Coletiva, \\ Universidade do Vale do Rio \\ dos Sinos, \\ São Leopoldo, Brasil. \\ Correspondência \\ J. S. Dias-da-Costa \\ Departamento de Medicina \\ Social, Faculdade de \\ Medicina, Universidade \\ Federal de Pelotas. \\ Av. Duque de Caxias 250, \\ Pelotas, $R S$ \\ 96030-002, Brasil. \\ episoares@terra.com.br
}

\section{Abstract}

This study assessed the quality of primary care in Pelotas, Rio Grande do Sul, Brazil, through preventable hospitalization rates (1995-2004). Preventable hospitalizations were defined as those related to the following diseases: diabetes mellitus, congestive heart failure, hypertension, chronic obstructive pulmonary disease, and vaccine-preventable diseases (polio, diphtheria, tetanus, whooping cough, and measles). Men and women from 20 to 59 years of age were included in the study. The proportion of preventable causes among hospital admissions was higher for women than for men. From 1995 to 2004 there was a decrease in preventable hospitalization rates. Even after direct standardization, analysis showed that admission rates in Pelotas were lower than for the State of Rio Grande do Sul as a whole. Preventable hospitalization costs decreased in parallel with the drop in hospital admission rates. The decrease in hospitalizations is consistent with improvement in primary health care. However, the findings may result from the system's financial model. Reimbursement for hospital procedures is low, which could induce the hospitals to curtail admissions.

Quality of Health Care; Primary Health Care; Hospitalization; Health Services
Juvenal Soares Dias-da-Costa 1,2

Laura Garcia de Borba 1

Michele Nunes Pinho 1

Moema Chatkin 1

\section{Introdução}

No Brasil, a implantação do processo de gestão plena do sistema de saúde coloca responsabilidades às prefeituras municipais com a efetividade dos cuidados em saúde, que são oferecidos de forma cada vez mais complexa. A partir da implantação do Sistema Único de Saúde (SUS) e com a municipalização dos estabelecimentos federais e estaduais, os serviços de saúde de natureza municipal experimentaram uma taxa geométrica de crescimento muito superior às demais unidades de saúde de outros níveis federativos 1. Com base em dados da pesquisa Assistência Médico-Sanitária do Instituto Brasileiro de Geografia e Estatística (IBGE), realizada em 1999, Viacava \& Bahia 2 verificaram que o crescimento dos estabelecimentos ambulatoriais sem internação na Região Sul do país foi de 1,1\%; porém, constataram que a rede ambulatorial era predominantemente pública, atingindo mais de $75 \%$ dos estabelecimentos.

Diversos autores têm alardeado a necessidade do uso da epidemiologia na organização de serviços 3,4,5 e na institucionalização da avaliação de programas e ações de saúde 6,7 , como forma de qualificar e de aperfeiçoar o sistema de saúde no Brasil.

Estudos epidemiológicos observacionais podem ser utilizados para as avaliações em saúde ${ }^{8}$. Tentativas de se avaliar acesso da população e qualidade dos cuidados da rede básica têm 
sido por intermédio da elaboração de estudos que descrevem as taxas de internações evitáveis 9,10,11. Essa avaliação parte de uma lista elaborada por uma comissão de especialistas sobre causas evitáveis de hospitalização ${ }^{9}$. E parte do princípio de que elevados números de hospitalizações evitáveis podem ser indicativos de problemas relacionados com a rede de atenção básica, seja na gestão, na quantidade insuficiente de serviços, na falta de medicamentos para o controle de doenças crônicas, dificuldades na oferta de recursos diagnósticos ou deficiências no manejo, no acompanhamento ambulatorial e no sistema de referência. Levando-se em conta, também, que tais hospitalizações também podem ser conseqüência da não aderência dos pacientes aos cuidados recomendados 12 .

A revisão da literatura, em que todos os estudos foram elaborados com fonte de dados secundários, mostrou que diferentes autores referem-se de forma variada a esse grupo de doenças e condições; alguns trabalhos denominam de hospitalizações preveníveis 10,11,13,14, outros consideram como hospitalizações por condições sensíveis aos cuidados ambulatoriais 15,16 ou como hospitalizações evitáveis 9,12,17. De qualquer forma, doenças como asma e insuficiência cardíaca congestiva, por exemplo, são condições para as quais o tratamento ambulatorial não pode evitar hospitalizações em todas as circunstâncias, entretanto, as doenças passíveis de controle por imunizações devem ser prevenidas em todos os casos 9,17. Assim como, pessoas portadoras de diabetes mellitus que receberam cuidados ambulatoriais adequados não devem ser hospitalizadas em condições de elevada severidade da doença 16. As internações de crianças e idosos foram excluídas pela possibilidade de essas condições se manifestarem de forma mais severa nessas faixas etárias. Em sistemas de saúde planejados para atingirem sua plena efetividade, a ocorrência de internações por essas condições deve atingir um percentual mínimo, pois se reconhece que quando os cuidados ambulatoriais são apropriados sob o aspecto clínico e propiciados no tempo correto podem ajudar a reduzir o risco de hospitalizações, seja por prevenir o início da doença ou condição, ou por manejar uma doença crônica 15. Parte-se do pressuposto teórico estabelecido por Campbell et al. 18 (p. 1.617) de que "a definição de qualidade do cuidado para populações depende da habilidade para acessar cuidados efetivos em bases eficientes e eqüitativas, buscando a otimização dos benefícios em saúde e o bem estar de toda a população". E do ponto de vista teórico, a efetividade de uma intervenção em saúde depende de cinco dimensões: da eficácia, da precisão diagnóstica, da aderência profissional, da aderência do receptor da ação e da cobertura 19 .

Pelotas é uma cidade situada ao sul do Estado do Rio Grande do Sul, tendo uma população estimada em 320 mil habitantes, em 2000. O Plano Municipal de Saúde descreveu uma extensa rede assistencial constituída por 48 unidades sanitárias, quatro ambulatórios de especialidades e cinco hospitais. A essa rede somam-se inúmeros serviços contratados. A rede de serviços ambulatoriais tem se expandido desde 1986 e cerca de $28 \%$ da população está coberta pelo Programa Saúde da Família (PSF). Estudos epidemiológicos demonstraram que a utilização de serviços básicos de saúde ficou facilitada após a criação e ampliação da rede ambulatorial 20,21. O número de hospitais permaneceu constante no período do estudo. O estágio de municipalização da saúde está implantado na forma de gestão plena do sistema.

Portanto, como uma tentativa de se avaliar a qualidade da rede de atenção básica realizouse estudo para verificar, mediante análise de tendência, algumas doenças classificadas como hospitalizações evitáveis em Pelotas, no período entre 1995 e 2004.

\section{Materiais e métodos}

O projeto de pesquisa foi aprovado pelo Comitê de Ética em Pesquisa da Faculdade de Medicina da Universidade Federal de Pelotas.

As causas de doenças consideradas como evitáveis estudadas foram: diabetes mellitus, insuficiência cardíaca, hipertensão arterial sistêmica, doença pulmonar obstrutiva crônica e doenças imunopreveníveis (constituídas por poliomielite, difteria, tétano, coqueluche e sarampo), nos indivíduos de 20 a 59 anos. Tais condições foram estabelecidas por comissões de peritos, para os indivíduos nessa faixa etária e têm sido utilizadas em estudos que avaliam qualidade da rede básica $9,10,11$.

Os códigos utilizados para doença pulmonar obstrutiva crônica entre 1995 e 1997 foram originados da Classificação Internacional de Doenças - 9a revisão (CID-9) 22 para bronquite crônica e bronquite não especificada, enfisema e asma (490-493). No período a partir de 1998, foram utilizados códigos da 10a revisão da classificação (CID-10) 23 para bronquite, enfisema, outras doenças pulmonares obstrutivas crônicas (J40-J43) e asma (J45). Os códigos para diabetes mellitus foram 181 na CID-9 e E10-E14 na CID-10; para hipertensão arterial sistêmica foram 401 (CID-9) e I10 e I11 (CID-10); para insuficiência cardíaca foram 428 (CID-9) e I50 (CID-10); para coquelu- 
che foram 033 (CID-9) e A37 (CID-10); para difteria foram 032 (CID-9) e A36 (CID-10); para tétano foram 037 (CID-9) e A35 (CID-10); para sarampo foram 055 (CID-9) e B05 (CID-10); para poliomielite foram 045 (CID-9) e A80 (CID-10).

Foram excluídas da análise as internações hospitalares por complicações da gravidez, parto e puerpério e por transtornos mentais.

Os dados de internações hospitalares em Pelotas estavam disponíveis na página da Internet do Departamento de Informática do SUS (DATASUS; http://www.datasus.gov.br) - morbidade e informações epidemiológicas/Sistema de Informações Hospitalares (SIH). Sendo possível obter informações sobre o número de internações segundo o local de residência, a faixa etária, o capítulo da CID-9 e CID-10 e o valor total pago pelas hospitalizações, desde o ano de 1995.

Os dados populacionais também estavam disponíveis na página da Internet do DATASUS, segundo faixa etária e sexo, desde 1979.

Para a análise, foram utilizados os seguintes indicadores: taxa bruta de internações evitáveis em Pelotas de 1995 a 2004 para as pessoas de 20 a 59 anos de ambos os sexos; taxa padronizada (pela população do Rio Grande do Sul em 2000) de internações evitáveis por idade e sexo em Pelotas de 1995 a 2004; taxa padronizada por idade e sexo de internações evitáveis no Rio Grande do Sul de 1995 a 2004; valor total pago pelas internações evitáveis em Pelotas de 1995 a 2004. As taxas de internações foram ajustadas por uma população padrão por meio do método direto de padronização. A padronização foi realizada tomando-se a população do censo do Rio Grande do Sul em 2000, realizado pelo IBGE. Os resultados expressam o número de internações que seriam esperadas na população padrão se as taxas por idade específica tivessem prevalecido. Esse procedimento anulou a influência da distribuição etária nas comparações entre as populações.

As significâncias das taxas brutas de internações evitáveis foram testadas pelo teste de tendência linear do programa Epi Info (Centers for Disease Control and Prevention, Atlanta, Estados Unidos) 24.

As estimativas das taxas padronizadas foram estabelecidas com base em seus intervalos de 95\% de confiança (IC95\%) 25 .

\section{Resultados}

Os percentuais de causas evitáveis em relação ao total de internações hospitalares entre as mulheres foram superiores aos encontrados no grupo masculino, em quase todos os anos. Foi observada uma diminuição dos percentuais de internações evitáveis tanto nos homens como nas mulheres. Entre os homens, constatou-se uma tendência ao crescimento até o ano de 1997, o percentual ficou oscilando entre 1998 e 2000, a partir de então foi decrescendo até 2004. Foi alcançada uma diminuição de 52,6\% na comparação entre o maior percentual $(9,7 \%)$ em 1997 e o menor (4,6\%) em 2004 (Tabela 1). Entre as mulheres, o percentual de causas evitáveis atingiu seu valor máximo em 1996, oscilou até 2000 , e depois também foi diminuindo até 2004. O percentual de diminuição constatado entre o maior valor - $13,5 \%$ em 1996 - e o menor - que também correspondeu a 4,6\% em 2004 - foi de 65,9\% (Tabela 2). Essa diminuição observada em ambos os sexos não foi produzida na diminuição de determinada causa, mas pelo conjunto das doenças analisadas.

Predominantemente, a análise por causas evitáveis mostrou que a insuficiência cardíaca foi a principal causa entre os homens em oito anos entre o total de períodos estudados (Tabela 1). Já entre as mulheres, a cada ano encontraram-se variações entre insuficiência cardíaca, diabetes mellitus e doença pulmonar obstrutiva crônica, alternando-se como maior percentual em relação total de internações (Tabela 2).

Ainda em relação às causas de internações evitáveis, deve-se destacar que apenas um caso de tétano foi constatado entre as mulheres em relação às doenças imunopreveníveis, enquanto 12 internações (11 casos de tétano e 1 caso de difteria) foram observadas entre os homens.

A análise mostrou tanto em homens como nas mulheres uma relação direta nas taxas de internações por 100 mil habitantes com o transcorrer da idade. Observou-se que as taxas nas mulheres sempre foram superiores às dos homens, exceto a do grupo etário compreendido entre 50 e 59 anos (Tabela 3).

Entre os homens, verificou-se que as taxas de internações foram se elevando até o ano de 1997, mantiveram-se estáveis até 2000 , e a partir de então foram decrescendo, e em 2003 e 2004 foram inferiores aos valores encontrados em 1995. A diferença percentual entre 1997 (maior taxa) e 2004 (menor taxa) foi de $-64,5 \%$.

De forma semelhante, a análise entre as mulheres mostrou um aumento nas taxas de internações entre 1996 e 1997, a partir daí as taxas foram semelhantes até 2000 , iniciando um declínio, observando-se que os valores alcançados em 2003 e 2004 foram inferiores aos de 1995. A diferença percentual entre a maior e menor taxa observada foi de $-65,7 \%$ (Tabela 3 ).

Tanto entre os homens como entre mulheres foram observados testes de tendência significativos ( $\mathrm{p}<0,00001)$ (Tabela 3$)$. 
Tabela 1

Percentual de internações por causas evitáveis entre os homens de 20 a 59 anos. Pelotas, Rio Grande do Sul, Brasil, 1995 a 2004.

\begin{tabular}{|c|c|c|c|c|c|c|c|c|c|c|}
\hline \multirow[t]{2}{*}{ Causas } & \multicolumn{2}{|c|}{1995} & \multicolumn{2}{|c|}{1996} & \multicolumn{2}{|c|}{1997} & \multicolumn{2}{|c|}{1998} & \multicolumn{2}{|c|}{1999} \\
\hline & $\mathbf{n}$ & $\%$ & $\mathbf{n}$ & $\%$ & $\mathbf{n}$ & $\%$ & $\mathbf{n}$ & $\%$ & $\mathbf{n}$ & $\%$ \\
\hline Diabetes & 54 & 2,2 & 92 & 2,6 & 66 & 1,7 & 24 & 0,6 & 25 & 0,6 \\
\hline Insuficiência cardíaca & 96 & 3,9 & 147 & 4,2 & 160 & 4,2 & 143 & 3,6 & 138 & 3,4 \\
\hline Hipertensão arterial sistêmica & 40 & 1,6 & 47 & 1,3 & 30 & 0,8 & 19 & 0,5 & 33 & 0,8 \\
\hline Doença pulmonar obstrutiva crônica & 25 & 1,0 & 47 & 1,3 & 110 & 2,9 & 125 & 3,2 & 157 & 3,8 \\
\hline Imunopreveníveis & 2 & 0,1 & 2 & 0,0 & 0 & - & 1 & 0,0 & 1 & 0,0 \\
\hline Total de doenças evitáveis & 217 & 8,8 & 335 & 9,5 & 366 & 9,7 & 312 & 7,9 & 354 & 8,7 \\
\hline Total de internações & 2.471 & - & 3.535 & - & 3.788 & - & 3.951 & - & 4.070 & - \\
\hline \multirow[t]{2}{*}{ Causas } & \multicolumn{2}{|c|}{2000} & \multicolumn{2}{|c|}{2001} & \multicolumn{2}{|c|}{2002} & \multicolumn{2}{|c|}{2003} & \multicolumn{2}{|c|}{2004} \\
\hline & $\mathbf{n}$ & $\%$ & $\mathrm{n}$ & $\%$ & $\mathbf{n}$ & $\%$ & $\mathrm{n}$ & $\%$ & n & $\%$ \\
\hline Diabetes & 58 & 1,5 & 72 & 2,1 & 57 & 1,8 & 49 & 1,9 & 41 & 1,3 \\
\hline Insuficiência cardíaca & 131 & 3,4 & 86 & 2,5 & 95 & 2,9 & 41 & 1,6 & 54 & 1,8 \\
\hline Hipertensão arterial sistêmica & 42 & 1,1 & 30 & 0,9 & 32 & 1,0 & 24 & 0,9 & 26 & 0,8 \\
\hline Doença pulmonar obstrutiva crônica & 97 & 2,5 & 81 & 2,4 & 55 & 1,7 & 26 & 1,0 & 20 & 0,7 \\
\hline Imunopreveníveis & 1 & 0,0 & 2 & 0,0 & 0 & - & 2 & 0,1 & 1 & 0,0 \\
\hline Total de doenças evitáveis & 329 & 8,6 & 271 & 8,0 & 239 & 7,4 & 142 & 5,6 & 142 & 4,6 \\
\hline Total de internações & 3.820 & - & 3.389 & - & 3.248 & - & 2.548 & - & 3.054 & - \\
\hline
\end{tabular}

Tabela 2

Percentual de internações por causas evitáveis entre as mulheres de 20 a 59 anos. Pelotas, Rio Grande do Sul, Brasil, 1995 a 2004.

\begin{tabular}{|c|c|c|c|c|c|c|c|c|c|c|}
\hline \multirow[t]{2}{*}{ Causas } & \multicolumn{2}{|c|}{1995} & \multicolumn{2}{|c|}{1996} & \multicolumn{2}{|c|}{1997} & \multicolumn{2}{|c|}{1998} & \multicolumn{2}{|c|}{1999} \\
\hline & $\mathrm{n}$ & $\%$ & $\mathbf{n}$ & $\%$ & $\mathrm{n}$ & $\%$ & $\mathbf{n}$ & $\%$ & $\mathrm{n}$ & $\%$ \\
\hline Diabetes & 73 & 2,8 & 170 & 4,3 & 128 & 2,9 & 36 & 0,7 & 44 & 0,9 \\
\hline Insuficiência cardíaca & 93 & 3,6 & 138 & 3,5 & 158 & 3,6 & 178 & 3,7 & 134 & 2,7 \\
\hline Hipertensão arterial sistêmica & 104 & 4,0 & 136 & 3,4 & 130 & 3,0 & 33 & 0,7 & 45 & 0,9 \\
\hline Doença pulmonar obstrutiva crônica & 52 & 2,0 & 89 & 2,2 & 143 & 3,3 & 191 & 3,9 & 187 & 3,8 \\
\hline Imunopreveníveis & 0 & - & 0 & - & 0 & - & 0 & - & 1 & 0,0 \\
\hline Total de doenças evitáveis & 322 & 12,5 & 533 & 13,5 & 559 & 12,7 & 438 & 9,0 & 411 & 8,3 \\
\hline Total de internações & 2.578 & - & 3.961 & - & 4.391 & - & 4.847 & - & 4.929 & - \\
\hline \multirow[t]{2}{*}{ Causas } & \multicolumn{2}{|c|}{2000} & \multicolumn{2}{|c|}{2001} & \multicolumn{2}{|c|}{2002} & \multicolumn{2}{|c|}{2003} & \multicolumn{2}{|c|}{2004} \\
\hline & $\mathbf{n}$ & $\%$ & $\mathbf{n}$ & $\%$ & $\mathbf{n}$ & $\%$ & $\mathbf{n}$ & $\%$ & $\mathbf{n}$ & $\%$ \\
\hline Diabetes & 130 & 2,8 & 136 & 3,0 & 133 & 3,1 & 85 & 2,5 & 70 & 1,5 \\
\hline Insuficiência cardíaca & 156 & 3,4 & 87 & 1,9 & 88 & 2,1 & 51 & 1,5 & 72 & 1,6 \\
\hline Hipertensão arterial sistêmica & 109 & 2,3 & 107 & 2,4 & 63 & 1,5 & 56 & 1,7 & 37 & 0,8 \\
\hline Doença pulmonar obstrutiva crônica & 135 & 2,9 & 109 & 2,4 & 75 & 1,8 & 30 & 0,9 & 31 & 0,7 \\
\hline Imunopreveníveis & 0 & - & 0 & - & 0 & - & 0 & - & 0 & - \\
\hline Total de doenças evitáveis & 530 & 11,4 & 439 & 9,8 & 359 & 8,5 & 222 & 6,6 & 210 & 4,6 \\
\hline Total de internações & 4.635 & - & 4.488 & - & 4.236 & - & 3.340 & - & 4.600 & - \\
\hline
\end{tabular}


A diminuição das taxas de internações evitáveis no Rio Grande do Sul não foi tão expressiva. Entre os homens, por exemplo, a menor taxa foi encontrada em 1995, e a diferença percentual foi de $-31 \%$. Entre mulheres para todo o estado, durante o período estudado, foi verificada uma diferença de $-35 \%$, entre o ano com a maior taxa (1997) e aquele com menor valor (2004). As análises de tendência temporal para o Estado do Rio Grande do Sul em ambos os sexos também mostraram testes de tendência linear significativos ( $\mathrm{p}<0,00001$ ) (Tabela 3).

Na comparação bruta das taxas de internação por 100 mil habitantes, constatou-se que as internações em Pelotas, em ambos os sexos, sempre foram inferiores às do Estado do Rio Grande do Sul (Tabela 3).

Mesmo após a padronização direta, a análise revelou que as taxas de internação em Pelotas foram durante todos os anos, para ambos os sexos, inferiores às observadas no Rio Grande do Sul, resultados confirmados pelas estimativas estabelecidas por intermédio dos intervalos de confiança (Tabela 4).

Em relação ao valor total pago pelas internações hospitalares, observaram-se valores crescentes até o ano de 1999. Deve-se destacar que, os valores alcançados em 1999, referentes ao to- tal de internações, foram aproximadamente três vezes maiores do que aqueles de 1995. A partir de 2000, os valores decresceram e voltaram a aumentar em 2004 (Tabela 5).

Os valores pagos pelas hospitalizações evitáveis acompanharam a queda observada nos percentuais e nas taxas de internações. Assim, em 1997, as internações evitáveis atingiram praticamente $15 \%$ dos recursos pagos pelas internações de Pelotas e em 2003 não alcançaram 5\% (Tabela 5).

\section{Discussão}

Os resultados do presente estudo mostraram uma diminuição das taxas de internações evitáveis no período estudado; contudo, à medida que o trabalho foi sendo elaborado, indagou-se se esse efeito foi conseqüência da qualidade da atenção básica.

O primeiro aspecto que deve ser discutido no presente trabalho é a escolha das condições consideradas como internações evitáveis. Torna-se impossível do ponto de vista clínico uma avaliação tão criteriosa realizada com base em dados secundários, que possa determinar verdadeiramente necessidade ou não de internação.

\begin{tabular}{|c|c|c|c|c|c|c|c|c|c|c|}
\hline Idade (anos) & 1995 & 1996 & 1997 & 1998 & 1999 & 2000 & 2001 & 2002 & 2003 & 2004 \\
\hline \multicolumn{11}{|l|}{$20-29$} \\
\hline Homens & 48,8 & 102,3 & 115,1 & 46,4 & 62,5 & 77,1 & 64,6 & 91,9 & 55,4 & 27,4 \\
\hline Mulheres & 104,6 & 268,6 & 315,8 & 142,3 & 78,2 & 223,4 & 233,9 & 186,1 & 116,5 & 107,9 \\
\hline \multicolumn{11}{|l|}{$30-39$} \\
\hline Homens & 132,7 & 216,3 & 237,5 & 149,4 & 164,6 & 166,1 & 86,8 & 77,4 & 59,6 & 29,5 \\
\hline Mulheres & 282,2 & 534,7 & 408,1 & 230,6 & 304,0 & 300,2 & 290,4 & 244,8 & 119,2 & 121,8 \\
\hline \multicolumn{11}{|l|}{$40-49$} \\
\hline Homens & 439,2 & 548,0 & 648,2 & 500,3 & 499,7 & 457,5 & 396,3 & 329,4 & 163,0 & 194,6 \\
\hline Mulheres & 506,6 & 674,1 & 905,0 & 701,2 & 547,2 & 763,9 & 526,3 & 428,7 & 249,6 & 247,0 \\
\hline \multicolumn{11}{|l|}{$50-59$} \\
\hline Homens & 847,5 & $1.273,3$ & $1.305,8$ & $1.363,5$ & $1.609,5$ & $1.251,7$ & $1.073,6$ & 897,5 & 546,6 & 588,4 \\
\hline Mulheres & 897,0 & $1.272,4$ & $1.241,7$ & $1.289,1$ & $1.281,0$ & $1.277,8$ & $1.056,7$ & 842,9 & 575,3 & 506,8 \\
\hline \multicolumn{11}{|c|}{ Total em Pelotas } \\
\hline Homens * & 285,7 & 430,2 & 469,7 & 396,0 & 444,1 & 400,4 & 328,1 & 286,6 & 168,5 & 166,8 \\
\hline Mulheres * & 378,3 & 616,4 & 644,3 & 499,3 & 463,1 & 578,0 & 476,4 & 385,8 & 236,1 & 221,1 \\
\hline \multicolumn{11}{|c|}{ Total em Rio Grande do Sul } \\
\hline Homens * & 403,5 & 512,7 & 539,8 & 547,9 & 584,7 & 572,8 & 526,4 & 503,3 & 441,3 & 428,0 \\
\hline Mulheres * & 552,0 & 617,6 & 680,0 & 562,3 & 644,8 & 669,6 & 583,5 & 597,6 & 519,3 & 441,6 \\
\hline
\end{tabular}

* Teste de tendência linear $p<0,00001$. 
Taxas de internações evitáveis padronizadas por idade e intervalos de $95 \%$ de confiança (IC95\%), para ambos os sexos, entre 20 e 59 anos, Pelotas, e no Estado do Rio Grande do Sul, Brasil, 1995 a 2004.

\begin{tabular}{|c|c|c|c|c|c|c|c|c|c|c|}
\hline & \multicolumn{10}{|c|}{ Taxa padronizada (IC95\%) } \\
\hline & 1995 & 1996 & 1997 & 1998 & 1999 & 2000 & 2001 & 2002 & 2003 & 2004 \\
\hline \multicolumn{11}{|l|}{ Pelotas } \\
\hline \multirow[t]{2}{*}{ Homens } & 299,5 & 398,4 & 476,0 & 409,6 & 443,1 & 407,6 & 367,5 & 306,6 & 192,3 & 176,4 \\
\hline & $(297,0-301,9)$ & $(395,7-401,1)$ & ) $(473,1-478,9)$ & $(406,8-412,3)$ & $(440,3-446,0)$ & $(405,0-410,1)$ & $(365,0-370,0)$ & $(304,5-308,8)$ & $(190,6-194,0)$ & $(174,7-178,1)$ \\
\hline \multirow[t]{2}{*}{ Mulheres } & 391,4 & 565,9 & 647,1 & 506,1 & 464,0 & 581,2 & 490,2 & 447,6 & 257,2 & 249,4 \\
\hline & $(389,6-393,1)$ & $(546,0-567,9)$ & ) $(644,8-649,3)$ & $(504,2-507,9)$ & $(462,4-465,7)$ & $(579,3-583,1)$ & $(488,6-491,9)$ & $(446,0-449,2)$ & $(256,0-258,3)$ & $(248,2-250,6)$ \\
\hline \multirow{2}{*}{\multicolumn{11}{|c|}{$\begin{array}{l}\text { Rio Grande } \\
\text { do Sul }\end{array}$}} \\
\hline & & & & & & & & & & \\
\hline \multirow[t]{2}{*}{ Homens } & 438,6 & 522,0 & 549,0 & 598,9 & 641,7 & 573,7 & 531,4 & 501,2 & 452,2 & 434,3 \\
\hline & $(438,1-439,2)$ & $(521,5-522,6)$ & ) $(548,4-549,6)$ & $(598,3-599,5)$ & $(641,1-642,4)$ & $(573,2-574,3)$ & $(530,8-531,9)$ & $(500,7-501,8)$ & $(451,7-452,7)$ & ) $(433,8-434,8)$ \\
\hline \multirow[t]{2}{*}{ Mulheres } & 579,5 & 623,7 & 685,9 & 715,9 & 736,4 & 681,2 & 636,0 & 591,5 & 531,6 & 479,5 \\
\hline & $(579,1-579,9)$ & $(623,3-624,0)$ & ) $(685,5-686,3)$ & $(715,5-716,3)$ & $(736,0-736,7)$ & $(680,8-681,5)$ & $(635,6-636,3)$ & $(591,1-591,8)$ & $(531,3-531,9)$ & $(479,2-479,7)$ \\
\hline
\end{tabular}

Tabela 5

Valor total pago pelas internações, valor pago pelas internações evitáveis e percentuais dos valores das internações evitáveis para ambos os sexos, na faixa etária entre 20 e 59 anos em relação ao total. Pelotas, Rio Grande do Sul, Brasil, 1995 a 2004.

\begin{tabular}{lccc}
\hline Ano & $\begin{array}{c}\text { Custo total de } \\
\text { internações (Reais) }\end{array}$ & $\begin{array}{c}\text { Custo de internações } \\
\text { evitáveis (Reais) }\end{array}$ & $\%$ \\
\hline 1995 & $5.856 .445,00$ & $760.024,10$ & 13,0 \\
1996 & $9.775 .236,00$ & $1.305 .060,00$ & 13,4 \\
1997 & $10.702 .510,00$ & $1.558 .233,00$ & 14,6 \\
1998 & $13.164 .596,00$ & $1.726 .985,00$ & 13,1 \\
1999 & $15.781 .116,00$ & $1.930 .284,00$ & 12,2 \\
2000 & $10.716 .652,00$ & $1.212 .685,00$ & 11,3 \\
2001 & $10.505 .861,00$ & $990.600,70$ & 9,4 \\
2002 & $11.204 .953,00$ & $961.485,70$ & 8,6 \\
2003 & $10.160 .436,00$ & $489.560,60$ & 4,8 \\
2004 & $12.275 .673,00$ & $665.206,80$ & 5,4 \\
\hline
\end{tabular}

Bittencourt et al. 26, em trabalho que levantou a produção científica das aplicações do SIH/SUS, apontaram como aspecto negativo que informações de duas condições diagnósticas pode ser um problema quando se usam dados do sistema para estudar doenças que são sub-registradas e as co-morbidades e complicações. Advertem também que a unidade de análise é a baixa hospitalar ao invés do indivíduo, assim múltiplas internações por um mesmo agravo podem levar a superestimativas do problema 15,16,26,27. Estudo realizado em 1986 no Rio de Janeiro, Brasil, para analisar a qualidade das informações contidas em banco constituído por dados dos formulários de Autorizações de Internações Hospitalares (AIH), mostrou índices kappa para insuficiência cardíaca congestiva, hipertensão essencial e diabetes mellitus de $0,793,0,725$ e 0,775 , respectivamente 28 . Outro estudo realizado posteriormente em Maringá, Paraná, Brasil, para avaliar a concordância de diagnósticos nas AIH, concluiu que se podem utilizar essas informações com algum grau de confiança 29 . Ainda outro estudo realizado no Rio de Janeiro mostrou qualidade do diagnóstico por infarto agudo do miocárdio pela AIH satisfatória, com percentual de confirmação elevado (91,7\%) 30.

O presente estudo também implica a falácia ecológica, ou seja, a impossibilidade de individualizar as informações. Aspectos importantes na determinação das internações hospitalares tais como condições sócio-econômicas 10,11,15,17,21,31, cor da pele $16,17,21$, local de residência (urbana ou rural) 16,17 , existência de fonte regular para consulta ambulatorial 10,12,15, presença de companheiro 16,27 e estado de saúde 15,16 não foram analisados. No Brasil, estudos com dados nacionais têm mostrado que nível sócio-econômico, sexo, idade, cor da pele, condições de saúde, existência de procura regular de serviços de saúde são fatores associados à utilização de serviços de saúde $32,33,34,35$.

A análise foi cuidadosa, idade e sexo são características naturalmente associadas ao uso de serviços hospitalares, em razão da sua influência biológica na morbimortalidade ${ }^{36}$. Existem inúmeros estudos mostrando que as mulheres utilizam mais serviços de saúde do que os homens 16,37 , incluindo a procura para fins preventivos 33 . 
Em Pelotas e no Rio Grande (Estado do Rio Grande do Sul), estudos de base populacional revelaram que as mulheres consultaram mais do que os homens 20,38. Estudo com dados provenientes da Pesquisa Nacional por Amostra de Domicílios (PNAD) de 1998 mostrou o aumento das internações hospitalares com o decorrer da idade 32 . Assim, estratificou-se a análise por sexo e isolou-se o possível efeito da constituição populacional ao se utilizar o método de padronização direta 39 . Os efeitos encontrados para sexo e idade confirmaram o acerto da análise.

A análise comparativa das taxas de internações evitáveis em Pelotas nesses dez anos demonstrou uma redução em relação ao tempo disponível para análise e quanto ao Estado do Rio Grande do Sul. Tal diminuição expressiva não pode ser justificada como aumento das condições de acesso, uma vez que a rede básica de saúde foi estabelecida há mais de vinte anos. Todavia, aconteceram mudanças estruturais importantes como o aumento da incorporação de recursos humanos na rede básica. Além disso, quando um município opta pela gestão plena do sistema deve apresentar órgão de controle, avaliação e auditoria, que pode pela sua criação diminuir a quantidade de internações evitáveis.

Entretanto, outros aspectos devem ser destacados nessa discussão, tentando entender a diminuição verificada nas taxas de internações evitáveis, especialmente aqueles relacionados ao financiamento do sistema de saúde no Brasil. Os procedimentos analisados são considerados como de média complexidade, cujos valores de pagamento por conta do SUS são baixos e podem estar direcionando os hospitais da cidade para diminuição da oferta de leitos para essas condições. Esse entendimento pode ser facilitado pelo estudo de Castro et al. 35 (p. 281), com dados da PNAD em 1998 que constataram " $a$ existência de efeito da oferta de serviços de saúde em modelos controlados por necessidades sugere indução ou repressão da demanda de serviços de saúde pela oferta". Os autores mostraram também que "quanto maior o número de leitos disponíveis, maior a chance do indivíduo se internar" 35 (p. 282). Ainda argumentam "por outro lado, esse efeito significa também que, quando a oferta for insuficiente, internações justificadas por necessidades de saúde podem deixar de ocorrer" 35 (p. 282). Desse modo, a redução nas internações no Estado do Rio Grande do Sul, e o efeito observado em estudo realizado no Estado do Rio de Janeiro, onde também foi mostrada a diminuição no número absoluto de internações em todas as áreas ${ }^{40}$, podem expressar uma tendência na redução das hospitalizações condicionada pela forma de pagamento.

Os valores totais pagos pelas hospitalizações evitáveis devem ser interpretados como possibilidades de economias no sistema local de saúde que, dirigidos para a atenção básica, poderiam aumentar a efetividade dos cuidados. A efetividade da atenção básica é de interesse para os políticos, planejadores e gestores em saúde. Pacientes submetidos a cuidados ambulatoriais de qualidade comprometida se apresentarão ao sistema de saúde com doença avançada, com uso mais freqüente dos serviços de emergência, estando mais propensos a necessitarem de cuidados mais caros e com, provavelmente, resultados menos favoráveis 16 .

Alguns autores têm manifestado a necessidade da validação desse tipo de estudo 13,15,17. Certamente, estudos posteriores, utilizando desenhos de casos e controles ou de coortes, possibilitarão conclusões mais válidas do que as utilizadas nesta investigação e poderão propiciar análises mais completas com ajustes de modelos complexos que envolvam as inúmeras variáveis que definem a necessidade de hospitalizações. Talvez, outra forma de acompanhamento e comparação das internações evitáveis seria a incorporação na análise de eventos-sentinela também de média complexidade que não fossem sensíveis aos cuidados primários tais como doenças cerebrovasculares e apendicites. Mesmo que as condições de Pelotas e do Estado do Rio Grande do Sul sejam diferentes das demais localidades do país, acredita-se que a monitorização contínua de taxas de admissão nos hospitais possam tornar-se uma condição facilitadora para a gestão de sistemas municipais de saúde. Os dados do DATASUS estão disponíveis e são de fácil acesso. Logo, o exame contínuo das taxas de admissão hospitalar para condições evitáveis pode ser um instrumento útil para monitorar o comportamento de um grupo comum de doenças, detectando - com um marcador indireto - condições de qualidade da rede de atenção básica, do comportamento hospitalar para doenças de média complexidade, podendo ainda estabelecer necessidades e proporcionar valiosas informações para a escolha de prioridades locais de saúde ou alocar recursos limitados entre comunidades 27 . 


\section{Resumo}

Realizou-se estudo para avaliar mediante taxa de internações hospitalares evitáveis a qualidade dos cuidados oferecidos pela rede básica de saúde em Pelotas, Rio Grande do Sul, Brasil, no período entre 1995 a 2004. Foram consideradas como internações evitáveis: diabetes mellitus, insuficiência cardíaca, hipertensão arterial sistêmica, doença pulmonar obstrutiva crônica e doenças imunopreveníveis (poliomielite, difteria, tétano, coqueluche, sarampo). Foram incluídos homens e mulheres de 20 a 59 anos. Os percentuais entre as mulheres foram superiores aos encontrados nos homens. Foi observada uma diminuição dos percentuais de internações tanto nos homens como nas mulheres no decorrer do período. Mesmo após a padronização direta revelou-se que as taxas de internação de Pelotas foram inferiores às do Rio Grande do Sul. Os custos das hospitalizações evitáveis acompanharam a queda observada nas taxas de internações. Aparentemente, a diminuição verificada nas taxas de internações evitáveis pode estar relacionada à qualificação dos serviços de atenção básica. Contudo, os resultados podem ser conseqüências do financiamento do sistema de saúde. Os valores de pagamento desses procedimentos são baixos e podem estar direcionando os hospitais a uma diminuição da oferta de leitos.

Qualidade da Assistência à Saúde; Atenção Primária à Saúde; Hospitalização; Serviços de Saúde

\section{Referências}

1. Costa NR, Pinto LF. Avaliação de programa de atenção à saúde: incentivo à oferta de atenção ambulatorial e a experiência da descentralização no Brasil. Ciênc Saúde Coletiva 2002; 7:907-23.

2. Viacava F, Bahia L. Oferta de serviços de saúde: uma análise da pesquisa Assistência Médico-Sanitária (AMS) de 1999. Brasília: Instituto de Pesquisa Econômica Aplicada; 2002.

3. Penna MLF. Reflexões sobre a epidemiologia atual. Physis (Rio J) 1997; 7:109-21.

4. Travassos C, Novaes HMD. Investigação e avaliação em serviços de saúde. Cad Saúde Pública 2004; 20 Suppl 2:S144-5.

5. Santos IS, Victora CG. Serviços de saúde: epidemiologia, pesquisa e avaliação. Cad Saúde Pública 2004; 20 Suppl 2:S337-41.

6. Hartz MA. Institucionalizar e qualificar a avaliação: outros desafios para a atenção básica. Ciênc Saúde Coletiva 2002; 7:419-21.

7. Felisberto E. Monitoramento e avaliação na atenção básica: novos horizontes. Rev Bras Saúde Matern Infant 2004; 4:317-21.

\section{Colaboradores}

J. S. Dias-da-Costa participou na elaboração do projeto, na coleta de dados, na análise dos dados e na redação do artigo. L. G. Borba e M. N. Pinho participaram na coleta, análise dos dados e na revisão do artigo. M. Chatkin participou da análise e redação do artigo.

8. Black N. Why we need observational studies to evaluate the effectiveness of health care. BMJ 1996; 312:1215-8.

9. Weissman JS, Gatsonis C, Epstein AM. Rates of avoidable hospitalizations by insurance status in Massachusetts and Maryland. JAMA 1992; 268:2388-94.

10. Bindman AB, Grumbach K, Osmond D, Komarony M, Vranizan K, Lurie N, et al. Preventable hospitalizations and access to health care. JAMA 1995; 274:305-11

11. Billings J, Anderson GM, Neuman LS. Recent findings on preventable hospitalizations. Health Aff 1996; 15:239-49.

12. Foland J. Avoidable hospitalizations: an indicator of inadequate primary care. http://www.dph. state.ct.us/oppe/brief2001.htm (acessado em 14/ Mar/2005).

13. Bluestein J, Hanson K, Shea S. Preventable hospitalizations and socioeconomic status. Health Aff 1998; 17:177-89. 
14. Culler SD, Parchman ML, Przbylsky M. Factors related to potentially preventable hospitalizations among elderly. Med Care 1998; 36:804-17.

15. Shi LY, Samuels M, Pease M, Bailey W, Corley EH. Patient characteristics associated with hospitalizations for ambulatory care sensitive conditions in South Carolina. South Med J 1999; 92:989-98.

16. Yuen EJ. Severity of illness and ambulatory caresensitive conditions. Med Care Res Rev 2004; 61:376-91.

17. Pappas G, Hadden WC, Kosak LJ, Fisher GF. Potentially avoidable hospitalizations: inequalities in rates between US socioeconomic groups. Am J Public Health 1997; 87:811-6.

18. Campbell SM, Roland MO, Buetow SA. Defining quality of care. Soc Sci Med 2000; 51:1611-25.

19. Castiel LD. Inefetividade e ineficiência: reflexões sobre a epidemiologia e os serviços de saúde de um estado de mal-estar social. Cad Saúde Pública 1990; 6:27-39.

20. Dias-da-Costa JS, Facchini LA. Utilização de serviços ambulatoriais em Pelotas: onde a população consulta e com que freqüência? Rev Saúde Pública 1997; 31:360-9.

21. Dias-da-Costa JS, Victora CG, Barros FC, Halpern R, Horta BL, Manzolli P. Assistência médica materno-infantil em duas coortes de base populacional no Sul do Brasil: tendências e diferenciais. Cad Saúde Pública 1996; 12 Suppl 1:S59-66.

22. Organização Mundial da Saúde. Manual da classificação estatística internacional de doenças, lesões e causas de óbitos - 9a conferência de revisão. São Paulo: Centro Brasileiro de Classificação de Doenças em Português; 1985.

23. Organização Mundial da Saúde. Classificação estatística internacional de doenças e problemas relacionados à saúde, 10a revisão. São Paulo: Centro Colaborador da OMS para a Classificação de Doenças em Português; 1995.

24. Rosenberg D. Trend analysis and interpretation: key concepts and methods for maternal and child health professionals. Rockville: Maternal and Child Health Bureau; 1997.

25. Altman DG, Machin D, Bryant TN, Gardner MJ. Statistics with confidence. Bristol: BMJ Books; 2000.

26. Bittencourt SA, Camacho LAB, Leal MC. O Sistema de Informação Hospitalar e sua aplicação na saúde coletiva. Cad Saúde Pública 2006; 22:19-30.
27. Billings J, Zeitel L, Lukomnik J, Carey TS, Blank AE, Newman L. Impact of socioeconomic status on hospital use in New York city. Health Aff 1993; 12:162-73.

28. Veras CMT, Martins MS. A confiabilidade dos dados nos formulários de Autorização de Internação Hospitalar (AIH), Rio de Janeiro, Brasil. Cad Saúde Pública 1994; 10:339-55.

29. Mathias TA, Soboll ML. Confiabilidade de diagnósticos nos formulários de autorização de internação hospitalar. Rev Saúde Pública 1998; 32:526-32.

30. Escosteguy CC, Portela MC, Medronho RA, Vasconcellos MTL. O Sistema de Informações Hospitalares e a assistência ao infarto agudo do miocárdio. Rev Saúde Pública 2002; 36:491-9.

31. Caper P. The microanatomy of health care. Health Aff 1993; 12:174-7.

32. Castro MSM, Travassos C, Carvalho MS. Fatores associados às internações hospitalares no Brasil. Ciênc Saúde Coletiva 2002; 7:795-811.

33. Pinheiro RS, Viacava F, Travassos C, Brito AS. Gênero, morbidade, acesso e utilização de serviços de saúde no Brasil. Ciênc Saúde Coletiva 2002; 7:687-707.

34 Sawyer DO, Leite IC, Alexandrino R. Perfis de utilização de serviços de saúde no Brasil. Ciênc Saúde Coletiva 2002; 71:757-76.

35. Castro MSM, Travassos C, Carvalho MS. Efeito da oferta de serviços de saúde no uso de internações hospitalares no Brasil. Rev Saúde Pública 2005; 39:277-84.

36. Hulka BS, Wheat JR. Patterns of utilization: the patient perspective. Med Care 1985; 23:438-60.

37. Travassos C, Viacava F, Pinheiro R, Brito A. Utilização dos serviços de saúde no Brasil: gênero, características familiares e condição social. Rev Panam Salud Pública 2002; 11:365-73.

38. Mendoza-Sassi R, Béria JU, Barros AJ. Outpatient health services utilization and associated factors: a population-based study. Rev Saúde Pública 2003; 37:372-8.

39. Lilienfeld AM, Lilienfeld DE. Foundations of epidemiology. New York: Oxford University Press; 1980.

40. Gouvêa CSD, Travassos C, Fernandes C. Produção de serviços e qualidade da assistência hospitalar no Estado do Rio de Janeiro, Brasil - 1992 a 1995. Rev Saúde Pública 1997; 31:601-17.

Recebido em 03/Mar/2007

Versão final reapresentada em 06/Nov/2007

Aprovado em 23/Nov/2007 